\title{
Sensing Spatial Coherence of Light with Metasurfaces
}

\author{
O. Buchnev' ${ }^{1}$, T. Frank ${ }^{1,2}$, T. Cookson ${ }^{2}$, M. Kaczmarek ${ }^{2}$, P. Lagoudakis ${ }^{2}$ and V. A. Fedotov ${ }^{1}$ \\ 1. Optoelectronics Research Centre and Centre for Photonic Metamaterials, University of Southampton, SO17 1BJ, UK \\ 2. School of Physics and Astronomy, University of Southampton, SO17 1BJ, UK
}

\section{0 words:}

We report on a discovery that homogeneous metallic non-diffracting metasurfaces of a certain type allow robust speckle-free discrimination between different degrees of the spatial coherence of light. The effect has no direct analogue in natural materials and has been previously unseen in metamaterials (and metasurfaces in particular). It results in a qualitative change of the optical response of metasurfaces, whereby their transmission (and reflection) spectra acquires different spectral components, depending on whether the nano-structures are illuminated with spatially coherent or incoherent light. This effect is robust and exceptionally strong (e.g., the resulting absolute change in transmission exceeds $50 \%$ ), which makes it immediately suitable for practical applications, such as optical metrology, imaging and communications. Among the metasurfaces that have been found to exhibit the new effect are planar metamaterials featuring a continuous periodic zigzag pattern. The reported samples were designed to operate in the near-IR part of the spectrum and composed of arrays of continuous zigzag nano-wires, as well as their inversion, i.e., continuous zigzag nano-slits, covering the area of $\sim 20 \times 20 \mu \mathrm{m}^{2}$. The measured data suggest that these apparently trivial metasurfaces, while non-diffracting, can indeed behave differently under spatially incoherent and coherent illumination. The systematic experimental investigation and rigorous theoretical analysis of this phenomenon (the results of which will be presented at the conference) reveal that the effect is underpinned by strongly non-local response of the metasurfaces. Its mechanism involves interference of light scattered via non-dispersive delocalised plasmon modes uniquely supported by the fabric of the metasurfaces.

\section{0 words:}

We report on a discovery that homogeneous metallic non-diffracting metasurfaces of a certain type respond differently to spatially coherent and incoherent light, enabling robust speckle-free discrimination between different degrees of coherence. The effect has no direct analogue in natural materials and has been previously unseen in metamaterials. It results in a qualitative change of the optical response of metasurfaces, whereby their spectra acquires different components, depending on whether the nano-structures are illuminated with spatially coherent or incoherent light. This effect is robust and exceptionally strong, which makes it immediately suitable for practical applications, such as optical metrology, imaging and communications. 